\begin{tabular}{|c|l|}
\hline Title & $\begin{array}{l}\text { Calculation method of reflectance distributions for computer-generated holograms using the finite difference time } \\
\text { domain method }\end{array}$ \\
\hline Author(s) & Ichikawa, Tsubasa; Sakamoto, Y uji; Subagyo, A gus; Sueoka, Kazuhisa \\
\hline Citation & $\begin{array}{l}\text { A pplied Optics, 50(34), H211-H219 } \\
\text { https://doi.org/10.1364/A O.50.00H211 }\end{array}$ \\
\hline Issue Date & 2011-12-01 \\
\hline Doc URL & http://hdl.handle.net/2115/52133 \\
\hline Rights & O2011 Optical Society of A merica \\
\hline Type & article \\
\hline File Information & ao-50-34-H211.pdf \\
\hline
\end{tabular}

Instructions for use 


\title{
Calculation method of reflectance distributions for computer-generated holograms using the finite-difference time-domain method
}

\author{
Tsubasa Ichikawa, ${ }^{*}$ Yuji Sakamoto Agus Subagyo and Kazuhisa Sueoka \\ Graduate School of Information Science and Technology, Hokkaido University, \\ North 14 West 9, Sapporo-shi 060-0814, Japan \\ *Corresponding author: ichikawa@ist.hokudai.ac.jp
}

Received 1 August 2011; revised 31 October 2011; accepted 8 November 2011; posted 9 November 2011 (Doc. ID 152129); published 2 December 2011

\begin{abstract}
The research on reflectance distributions in computer-generated holograms (CGHs) is particularly sparse, and the textures of materials are not expressed. Thus, we propose a method for calculating reflectance distributions in CGHs that uses the finite-difference time-domain method. In this method, reflected light from an uneven surface made on a computer is analyzed by finite-difference time-domain simulation, and the reflected light distribution is applied to the CGH as an object light. We report the relations between the surface roughness of the objects and the reflectance distributions, and show that the reflectance distributions are given to CGHs by imaging simulation. (c) 2011 Optical Society of America

OCIS codes: $\quad 090.0090,090.1760$.
\end{abstract}

\section{Introduction}

A computer-generated hologram (CGH) is a technique for displaying ideal three-dimensional (3D) images. CGHs are made by simulating the diffusion, diffraction, and interference of light waves from virtual objects created on a computer. One advantage over normal holography is that the recorded object need not exist. Thus, making a hologram that records virtual objects is possible. In making a normal hologram, extensive equipment, such as optical systems and a darkroom, are needed for the recording. Thus, enormous time and effort are required, and risk is involved. It is possible to makpe a CGH by printing the interference pattern to film or displaying it in a spatial light modulator.

A CGH is one of several 3D display techniques, but there are some problems because a CGH deals with $3 \mathrm{D}$ objects and calculation in the optical range. In particular, enormous computation time and a lack of rendering techniques to display images of a quality

0003-6935/11/34H211-09 $\$ 15.00 / 0$

(C) 2011 Optical Society of America similar to that in the field of computer graphics (CG) are major issues. To decrease the calculation time, many methods have been proposed. For example, there are fast calculation methods using a graphics processing unit or a special-purpose computer $[1,2]$. Fast calculation algorithms, such as a method that uses a lookup table, and a technique that uses 3D Affine transforms of basic object light have also been reported $[3,4]$.

Research on rendering techniques to display realistic images have also been reported. A method of hidden-surface removal to deal with invisible parts of an object by crossing among objects has been described [5]. A method of shading the object and mapping that expresses patterns by pasting images to the object surface was proposed in [6]. Objects constructed of polygons have the problem that an object surface becomes angular unless patches become sufficiently small. Thus a smooth shading in which a patch is expressed as a curved surface has been proposed [7]. So methods for expressing hidden-surface removal, shading, smooth shading, and texture mapping have already been developed. 
However, research on reflectance distributions to express each material of object surfaces or surface roughness are scarce. The texture of a surface appears like plaster with the conventional method. In the field of CG, the texture of an object is expressed by selecting a reflection model that is suitable for each material and making luminance distributions. In CGHs, expressing complex reflectance distributions is difficult because phase information is needed in addition to luminance. To calculate the reflection of a light wave accurately, it is necessary to consider the reflection from a complex surface structure. The appearance of multiple reflections on a surface structure is a considerable problem.

Thus, we propose a calculation method to add reflectance distributions for CGHs by using a finitedifference time-domain (FDTD) method. The FDTD method is an electromagnetic field analysis method that analyzes accurately the reflection of light waves from a surface structure [8]. This paper describes a method in which reflected light obtained by FDTD is used as a surface function in CGHs. It is confirmed that a surface structure made on a computer gives a result similar to actual objects by comparing the specular reflectance. The result of imaging simulations confirms that various reflectance distributions are given at some surface roughness.

\section{Calculation Method of CGHs}

Here, we describe the process of making a CGH. When a CGH is made, the creation of virtual objects, propagation of the object light, addition of the reference light, and calculation of the interference pattern are carried out on a computer.

There are two methods to make a virtual object on the computer. One is the point light method [9]. Virtual objects are defined as aggregates of a point light source, and then propagation of light waves is calculated from each point light source to a hologram plane. Although it is advantageous for making arbitrary object shapes, there are problems, such as the enormous computation time and difficulty of dealing with reflections on the object surface.

Another method composes the object surfaces by using planes called patches. It takes a shorter computation time than the point light method by making objects with a patch model. It is also easy to consider reflectance distributions because object surfaces are treated as a plane. Hence, we selected a modeling method using a patch model for this study.

There are some propagation methods for light waves from virtual objects. Fresnel transform and Fraunhofer transform method using fast Fourier transform (FFT) calculate the fast propagation of light waves. In these methods, there are approximation errors and restrictions of the propagation distance because the approximation is used to determine the distance between planes. The angular spectrum (AS) method is another well-known calculation method [10]. A calculation method to correspond to propagation between inclination planes and to coun- teract the restriction of the propagation distance in the AS method has been proposed [11,12].

The two methods described above cannot calculate the propagation if the sampling pitch between planes is different. We need a method that propagates reflected light distributions obtained by FDTD analysis for this study. The sampling pitches of reflected light from FDTD analysis are far smaller than current output devices. Thus, we use the shifted-Fresnel method, which calculates the propagation between planes having different sampling pitches [13]. This method also calculates propagations between off-axis planes.

The shifted-Fresnel method is an extension of the calculation method using Fresnel transform. The coordinates on the bottom-left corner of the object plane and the hologram plane are, respectively, assumed to be $\left(x_{0}, y_{0}\right)$ and $\left(x_{0}^{\prime}, y_{0}^{\prime}\right)$, and the sampling pitches are $(\Delta x, \Delta y)$ and $\left(\Delta x^{\prime}, \Delta y^{\prime}\right)$, as shown in Fig. 1 . The hologram plane $x^{\prime}-y^{\prime}$ is located away from the o $\bar{b}$ ject plane $x-y$, and shifts horizontally. The coordinates of each plane are defined by the following equations:

$$
\begin{array}{ll}
x_{m}=x_{0}+m \Delta x, & y_{n}=y_{0}+n \Delta y, \\
x_{p}^{\prime}=x_{0}^{\prime}+p \Delta x^{\prime}, & y_{q}^{\prime}=y_{0}^{\prime}+q \Delta y^{\prime} .
\end{array}
$$

Here, $(m, n)$ and $(p, q)$ are, respectively, the index of pixels of an object plane and a hologram plane. The Fresnel transform is discretized by using Eqs. (1) and (2). The light distribution of hologram plane $d$ away from the object plane is calculated using the following equation:

$$
\begin{aligned}
U_{d}(m, n)= & (i \lambda d)^{-1} \exp (i k d) \exp \left[i \pi\left(x_{m}^{2}+y_{n}^{2}\right) / \lambda d\right] \\
& \cdot \exp \left[-i 2 \pi\left(x_{0}^{\prime} m \Delta x+y_{0}^{\prime} n \Delta y\right) / \lambda d\right] \\
& \times \sum_{p=0}^{P-1} \sum_{q=0}^{Q-1}\left(\left[u(p, q) \exp \left[i \pi\left(x_{p}^{\prime 2}+y_{q}^{\prime 2}\right) / \lambda d\right]\right.\right. \\
& \cdot \exp \left[-i 2 \pi\left(x_{p}^{\prime} x_{0}+y_{q}^{\prime} y_{0}\right) / \lambda d\right] \\
& \left.\cdot \exp \left[-i 2 \pi\left(\Delta x^{\prime} \Delta x p m+\Delta y^{\prime} \Delta y q n\right) / \lambda d\right]\right) .
\end{aligned}
$$

Here, $\lambda$ is the wavelength, and $P$ and $Q$ are, respectively, the number of pixels on each side of the object plane. This method is restricted in that the number

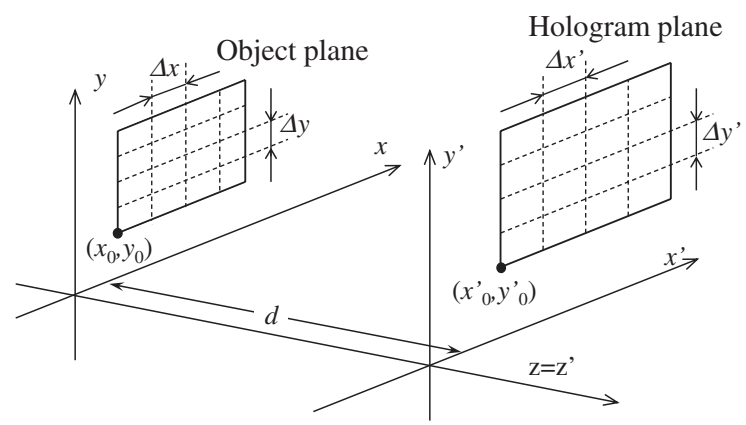

Fig. 1. Shifted-Fresnel diffraction geometry. 
of pixels in the object plane and hologram plane must be the same.

Equation (3) is transformed into the form of a convolution. $\bar{A}$ discrete convolution that uses three FFTs can be computed by applying the convolution theorem.

Finally, hologram data are calculated from the interference pattern by adding reference light to the light distribution of the hologram plane. The hologram is generated by outputting the hologram data to a device such as a SLM or sensitive materials.

\section{Reflectance Distributions}

\section{A. Reflectance Distributions}

In this section, we explain reflectance distributions before describing our proposed method. Reflectance distributions are features that express how the light is reflected when light from a light source enters an object surface. Thus, reflectance distributions indicate the distributions of luminance on an object surface and express the textures and materials of objects. Characteristics of reflectance distributions are divided into diffuse and specular reflections.

A diffuse reflection and a specular reflection are, respectively, shown in Figs. 2(a) and (b). In Fig. 2(a), a ray from the light source diffuses at random on an object surface. The intensity distributions of the reflected light form a semicircle having a constant strength that centers on the incident point. In contrast, in a specular reflection, the reflected light advances in a constant direction toward wherever the incident light reflects. Therefore, the reflectance distributions of specular reflection tend to increase in a specular direction [Fig. 2(b)].

\section{B. Conventional Method}

In $\mathrm{CGH}$, reflectance distributions are expressed by adding distributions of phase differences to complex amplitude distributions of object surfaces. When there is no phase difference, reflectance distributions become a perfect specular reflection because reflected light advances in a constant direction. This causes some problems. The edges of an object surface are very bright and prevent observers from seeing the object and, thus, only part of an object can be seen.

To diffuse the light wave of an object, a method is used that mainly adds white noise to light waves of object surfaces as distributions of phase differences [14]. In this method, a light wave of an object surface advances in any direction and reflectance distributions become a perfect diffuse reflection.

Other reflectance distributions can be created by controlling the ratio of specular and diffuse values $[15,16]$. In the field of CG, there are reflection models that formulate the specular reflection and the diffuse reflection. Methods that apply these reflection models to CGH have been proposed. In [15], the CookTorrance model is selected as a reflection model. The reflectance distributions are given by convolving

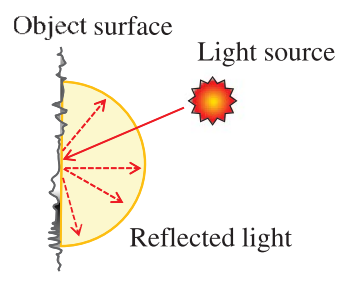

(a) Diffuse reflection.

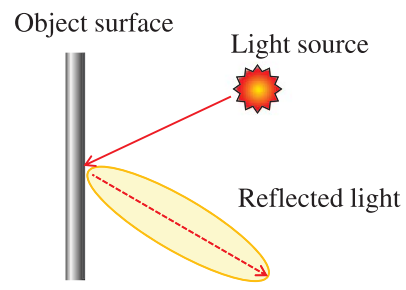

(b) Specular reflection.

Fig. 2. (Color online) Reflectance distributions.

the objective reflection with the propagation function. This method expresses the reflections only for metallic material because the Cook-Torrance model is suited for metallic objects.

Another method that uses the Torrance-Sparrow model and the Blinn model has been proposed [16]. In these reflection models, the object surface is assumed to be aggregate of microfacets, and the reflection is calculated by using geometric optics.

However, it is difficult for these methods to express complicated reflections, such as multiple reflections on a complex surface structure, subsurface scattering, shadowing, and masking. Moreover, differences of material, such as metal, plastic, wood, and glass, cannot be expressed. Therefore, we propose a method for calculating reflectance distributions by obtaining reflected light from FDTD analysis.

\section{Proposed Method}

The proposed method creates a CGH by the following process.

1. Make the surface structures of the object on a computer.

2. Analyze the electromagnetic field in the analysis area by the FDTD method, and obtain the distribution of reflected light.

3. Propagate the distributions of reflected light obtained by FDTD analysis to the hologram plane by the shifted-Fresnel method.

This method has some advantages. By using FDTD, our method enables reflections from any material and shape to be analyzed. Furthermore, multiple reflections on a complex surface structure, subsurface scattering, shadowing, and masking are computable. In addition, when another object is used, recalculation of the reflected light is unnecessary because it is possible to preserve reflected light obtained by the FDTD analysis. Moreover, it is easy to control the scattering angle by changing the 
surface roughness of the object surface. The detailed flow of the proposed method is described in the following subsections.

\section{A. Making the Surface Structure}

First, the surface structure of the object is made on a computer before FDTD analysis. We used two construction methods. One computationally makes a surface structure that has similar roughness to the actual object in consideration of surface roughness. The other method does modeling on a computer by using surface structure data obtained by atomic force measurement (AFM) of actual objects in order to compare with a surface roughness made by a former method.

\section{A.1. Making the Surface Structure Considering Surface Roughness}

When the structure of a surface is made, parameters of surface roughness established by ISO 4278 are used to obtain comparable roughness to the actual object. The basic parameters are the maximum height of the surface $R y$, the average length of the periodic structure $R a$, and the average value of roughness $R S m$ (Fig. 3). When the roughness curve is $f(x)$, each parameter is defined by the following equations:

$$
\begin{gathered}
R y=\max (f(x))-\min (f(x)), \\
R a=\frac{1}{l} \int_{0}^{l}|f(x)| \mathrm{d} x, \\
R S m=\frac{1}{m} \sum_{i=1}^{m} X s i .
\end{gathered}
$$

Therefore, the curved surface of the object $F(x, y)$ is expressed by the following equation that combines the sine curves:

$$
F(x, y)=A(x, y) \cdot R y \cdot \sin \left(\frac{2 \pi}{R S m} x\right) \sin \left(\frac{2 \pi}{R S m} y\right) .
$$
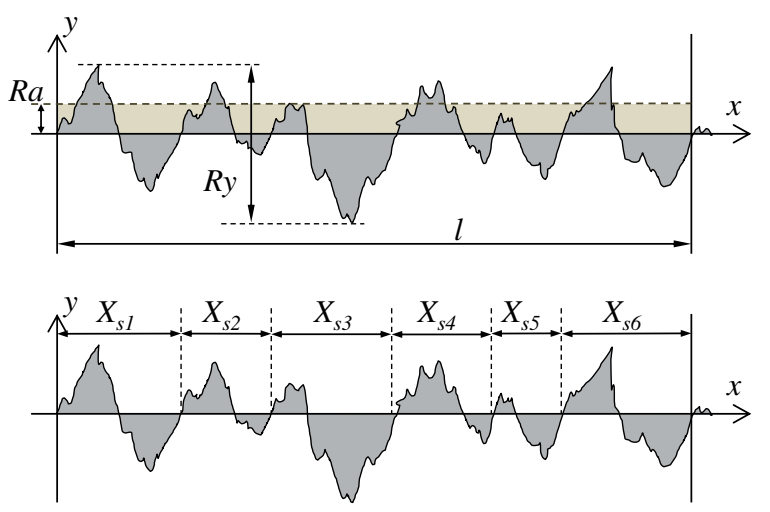

Fig. 3. (Color online) Surface roughness.
Here, $A(x, y)$ give the amplitude irregular values to prevent the amplitude of a surface structure from having a constant value. $A(x, y)$ are assigned to each periodic unevenness, and have uniformly values of 0 to 1 . Then, the average length of periodic structure $R a$ becomes $R y / 4$. One example of the surface structure made by Eq. (7) is shown in Fig. $\underline{4}$.

\section{A.2. Measurement of the Surface Structure with $A F M$}

Actual object surfaces are measured by AFM to make surface structures for comparison with Subsection 4.A.1. AFM is a kind of scanning probe microscope that obtains the surface structure by detecting the atomic force between the sample and the probe. The minute asperity of a surface structure is measured because the spatial resolution of an AFM is in the nano domain. We prepared three kinds of samples; iron, copper, and aluminum (Fig. 5). They were polished with sandpaper in advance to have varying roughness.

Figure 6 shows 3D images of surface structures measured by AFM. The average values of roughness $R a$ gauged by the AFM are in the captions. The figures confirm that the object surface has a complex structure that influences the reflection.

\section{B. FDTD Analysis}

Next we discuss how to apply the FDTD method to CGH. Ideally, the light wave distributions on the hologram plane would be calculated more accurately by propagating the light from whole objects to the hologram plane with the FDTD method. However, this is not practical because enormous amounts of memory and computation time are necessary. It is thus necessary to consider how to choose an analysis area that is appropriate for a CGH.

The FDTD method is effective at analyzing the scattering problem for a minute structure. When free-space propagation of reflected light to a distant hologram plane is calculated after light reflects on the object surface, there is no advantage in the calculation cost compared with other methods. Therefore, we analyzed only the neighborhood of a surface in this study and obtained the reflected light near the surface as the light distribution of an object surface.

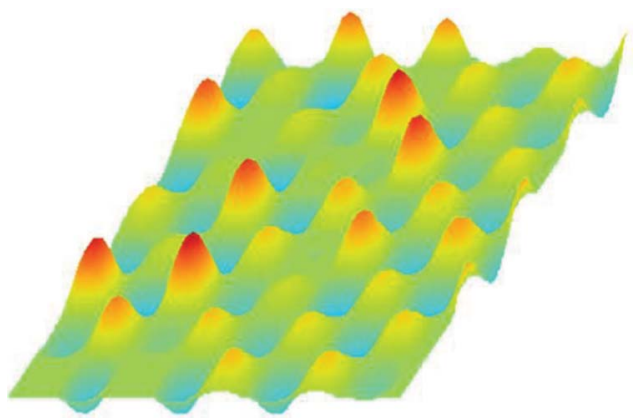

Fig. 4. (Color online) Uneven surface made by the method in Subsection 4.A.1. 


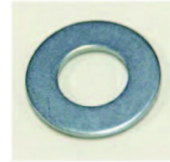

(a) Iron.

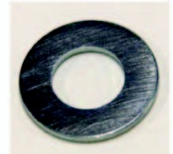

(b) Copper.

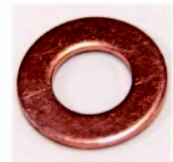

(c) Aluminum.
Fig. 5. (Color online) Samples.

However, the necessary memory and computation time become huge if reflection on the whole reflection plane is analyzed using the FDTD method, even if the depth of the analysis area is shortened. A part of the object surface structure is taken, and the FDTD analysis is done by directing the incident light perpendicular to the surface, as shown in Fig. 7. In this paper, the continuous wave shown by the following equation is used because only an analysis of the single wavelength is necessary:

$$
y(n \cdot \Delta t)=C \sin (2 \pi f \Delta t n) .
$$

Here, $C$ is amplitude, $f$ is frequency, $\Delta t$ is the width of the time step, and $n$ denotes the number of time steps.

Incident light is entered perpendicularly to the object surface from the $z$ direction. Walls made of a per-

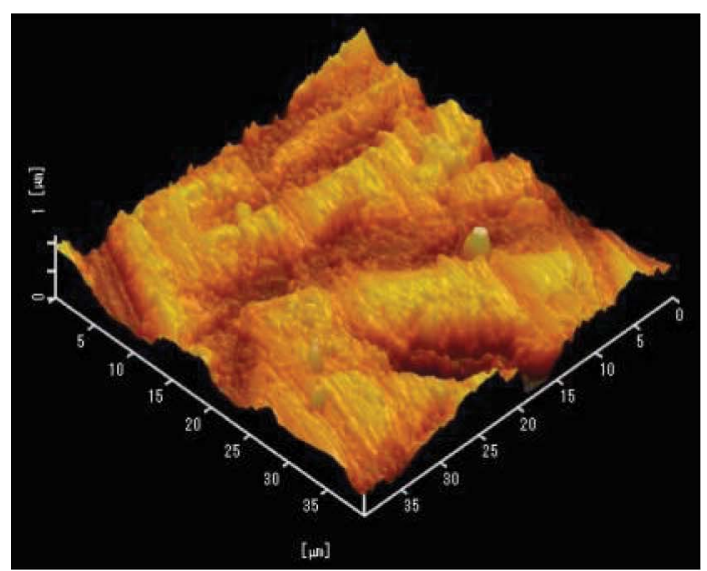

(a) $R a=0.2468[\mu \mathrm{m}]$.

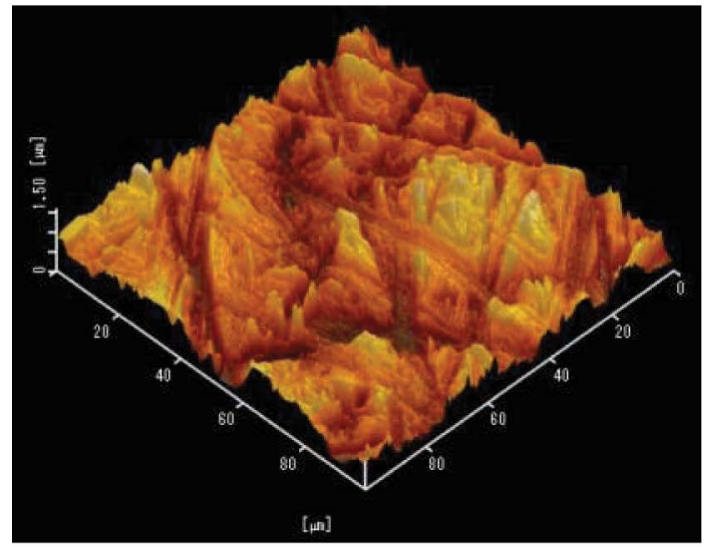

(b) $R a=0.1358[\mu \mathrm{m}]$.

Fig. 6. (Color online) 3D images of surface structure.

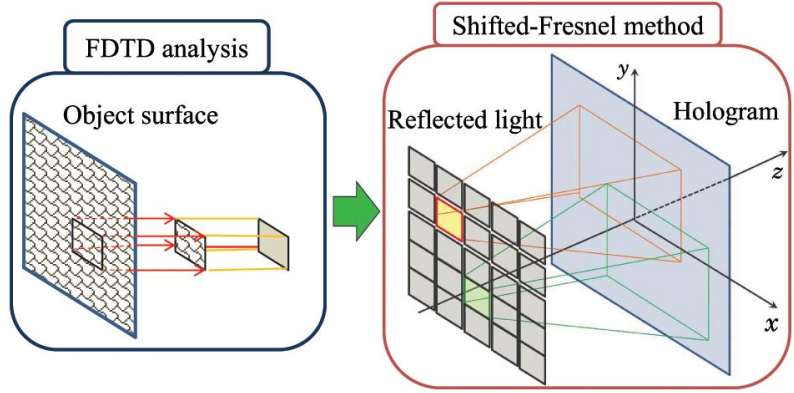

Fig. 7. (Color online) Calculation of reflection from the minute surface.

fect conductor are set at boundary planes in the $x$ and $y$ directions of the analysis area. If there are no perfect conductor walls, the scattering phenomenon becomes free-space propagation, and reflected light goes out of the boundary plane. Thus, when the patches of reflected light obtained by FDTD analysis are lined up to make a large surface, the reflected light does not reach the adjoined patches, as shown in Fig. 8(a). This leads to the problem that only the center of the light distribution tends to become strong. Reflection occurs on the boundary planes when walls made of a perfect conductor are set on the boundary planes, as shown in Fig. 8(b). The reflected light at perfect conductor walls is treated as an artificial diffuse light from outside of the boundary.

The parameters of the FDTD analysis are shown in Table 1 . Although the cell size is $0.05[\mu \mathrm{m}]$, it is necessary to model with the smaller cell in order to express fine structures on the surface correctly. So we used a local subgridding method that divides specified cells into smaller cells [17]. In this paper, fine structures are expressed by dividing the cell that contains the object surface. The size of divided cells is $0.005[\mu \mathrm{m}]$. The wavelength of light passing through a medium generally becomes shorter than in vacuum. So cell size inside the material must be smaller. However, in optical frequencies, the skin depths of the materials that we used are very low. Therefore, the area into which light invades is calculated only in divided cells near the object surface by the local subgridding method. In this analysis, only the scattered waves are required. It is necessary to subtract the incident electromagnetic field from calculation results. Then we conducted the FDTD analysis for the scattered field that calculates directly the scattered field. And here, the calculation time is the mean time until the reflection phenomenon reaches the stationary state. The reflection phenomenon shows various behaviors as surface roughness becomes deep and complicated, and the calculation time until obtaining a light wave in the stationary state lengthens.

\section{C. Calculation of Hologram Data}

In this study, the reflected light distributions obtained by FDTD analysis were stored in the database once. Reflected light distributions are treated as light waves on an object surface. When a large object is made in a virtual space for $\mathrm{CGH}$, the reflected light 


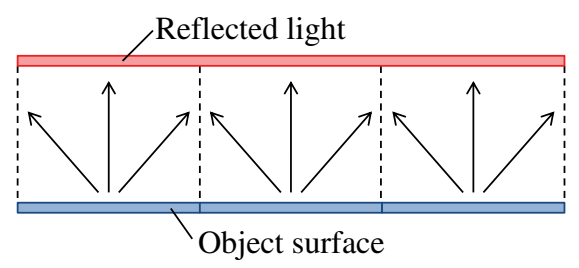

(a) Radiation to free-space.

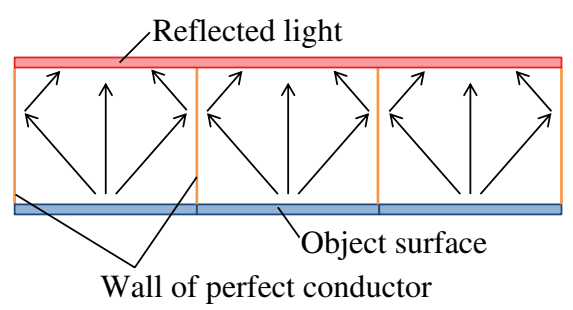

(b) Half closed region enclosed with perfect conductor.

Fig. 8. (Color online) Alignment of reflected light.

distributions are lined up along an object surface. The light wave of a large object at a hologram plane is calculated by conducting propagation from each patch that composes the large object to a hologram plane. This propagation process is conducted by the shifted-Fresnel method described in Section 2. The sampling pitches of reflected light distributions obtained by FDTD analysis are far smaller than the output devices. The shifted-Fresnel method obtains only light wave distributions of coarse resolution of a specific direction. So the computation time is comparatively short. Objects made of various materials are expressed in a CGH by preserving the reflected light distributions of the various materials and surface structures beforehand.

\section{Experiment}

\section{A. Computer Simulation}

We first conducted computer simulations to confirm whether the reflectance distributions obtained by the proposed method express the differences of materials and surface roughness. In this experiment, the reflected light of small patch was propagated to a hologram plane $1 \mathrm{~cm}$ away (Fig. 9). Then the specular reflectance that indicates how much light propagates in the specular direction is calculated. The specular reflectance is given by the following equation:

$$
R=\sum^{X} \sum^{Y} \frac{\left|u^{\prime}\left(x^{\prime}, y^{\prime}\right)\right|^{2}}{I_{0}(x, y)}
$$

Table 1. Setup Parameters for FDTD Analysis

$\begin{array}{ll}\text { Number of cells }(x, y, z) & 800 \times 800 \times 200 \text { [cells] } \\ \text { Cell size } & 0.05[\mu \mathrm{m}] \\ \text { Real size of analysis area } & 40 \times 40 \times 10[\mu \mathrm{m}] \\ \text { Calculation time } & 9[\mathrm{~h}]\end{array}$

Here, $I_{0}(x, y)$ is the light-wave intensity of the incident light in the FDTD analysis. The experiment parameters are shown in Table 2 . To make the surface structures, we used the two methods described in Subsections 4.A.1 and 4.A.2.

\section{A.1. Surface Structure Made by Method in Subsection 4.A.1}

Here, specular reflectances of surface structures made by the method described in Subsection 4.A.1 are investigated. Four kinds of materials are used: a perfect conductor, copper, iron, and black lead.

The relation between the specular reflectance of each material and the surface roughness $R a$ is shown in Fig. 10. In Fig. 10(a), the specular reflectance of the perfect conductor, copper, and iron are shown, and that of black lead is shown in Fig. 10(b). These graphs confirm that the specular reflectance of any material decreases as surface roughness increases. This is because light has a wider scatter angle as the surface becomes rougher, so the light wave does not fully reach to the hologram plane.

The specular reflectance of copper is confirmed to be similar to that of a perfect conductor from Fig. 10(a). This is because the electrical characteristics of copper in the wavelength used for the experiment are very near those of the perfect conductor. The specular reflectance of iron is lower than those of copper and the perfect conductor for all surface roughness. Regardless of surface roughness, the optical absorptance of iron is high compared with other metals, so iron has a semidull luster.

Figure 10(b) shows the specular reflectance of black lead; the scale interval of the ordinate axis is different from Fig. 10(a). The specular reflectance of black lead also decreases as the surface roughness increases. However, reconstructed images are not observed because the optical absorptance of black lead is very high. From these results, the differences between magnetic material and a dielectric substance that cannot be expressed by the conventional method are shown to be expressible by our method.

\section{A.2. Surface Structure Measured by $A F M$}

Specular reflectances of surface structures measured by AFM are shown in Fig. 11. These graphs confirm

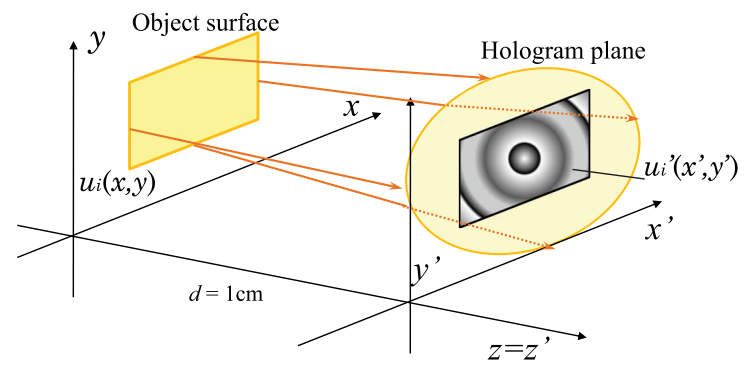

Fig. 9. (Color online) Experiment geometry. 
Table 2. Setup Parameters for Computer Simulation

Object Size

Number of pixels

Input sampling pitch

Output sampling pitch

Wavelength

Propagation distance
$200 \times 200[\mu \mathrm{m}]$

$4096 \times 4096$ [pixels]

$0.05 \times 0.05[\mu \mathrm{m}]$

$0.05 \times 0.05[\mu \mathrm{m}]$

$632[\mathrm{~nm}]$

$0.01[\mathrm{~m}]$

that the specular reflectance decreases as the surface roughness increases, as in Subsection 5.A.1.

Theoretical values of normal incidence reflectivity to a mirror surface are $97.5 \%$ (copper), $91.2 \%$ (aluminum), and $56.3 \%$ (iron) at the wavelength, and are shown in Fig. 11 by the cross marks. Approximate curves of the exponent function were added to these graphs. The $y$ intercepts of the approximate curves are reflectivities of a perfect specular surface. Each $y$ intercept in Fig. 11 is nearly equal to the theoretical values. Therefore, variation of the specular reflectance due to differences of materials is expressed by the proposed method.

To compare specular reflectances of a surface structure measured by AFM and made by Eq. 7, both the specular reflectances of copper and iron produced by each method are shown in Fig. 12. According to Fig. 12, it is confirmed that a surface structure made by Eq. 7 makes a result similar to that of a surface structure measured by AFM. However, the specular reflectances by AFM are a little bit lower than those by Eq. 7 . It is presumed that the scatter angle be-

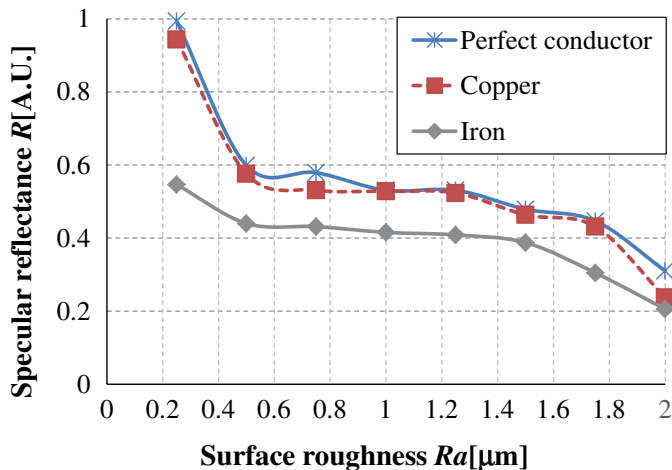

(a) Metallic substances.

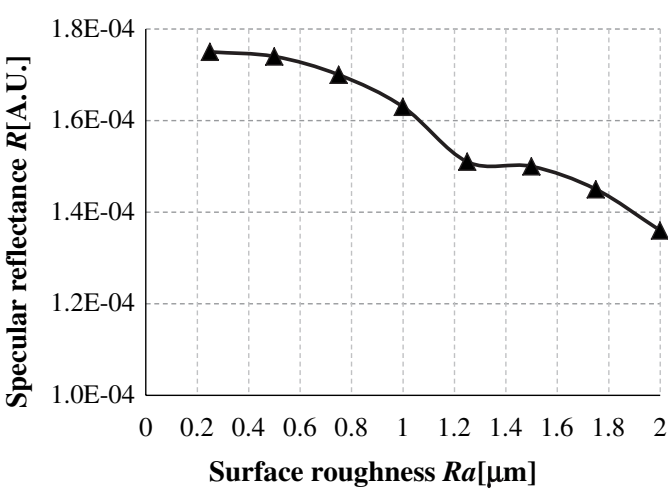

(b) Black lead.

Fig. 10. (Color online) Specular reflectance. comes wider due to the minute surface structure that Eq. 7 cannot express.

\section{B. Imaging Simulation}

We confirmed that, with the proposed method, it is possible to give reflectance distributions for a hologram by observing the $3 \mathrm{D}$ images of the imaging simulation. In this experiment, the reflected light distributions are applied to the cylindrical model shown in Fig. 13(a), and the light waves of the object are propagated to the hologram plane. Parameters of this experiment are shown in Table 3. A surface structure of copper measured by AFM is used.

Figure 13(b) is an image where the reflectance distribution of the perfect diffuse reflection by the conventional method was added by using the random phase method. Thus, the whole image shows the same luminance. Figures 13(c) and 13(d) have reflectance distributions from different surface roughness.

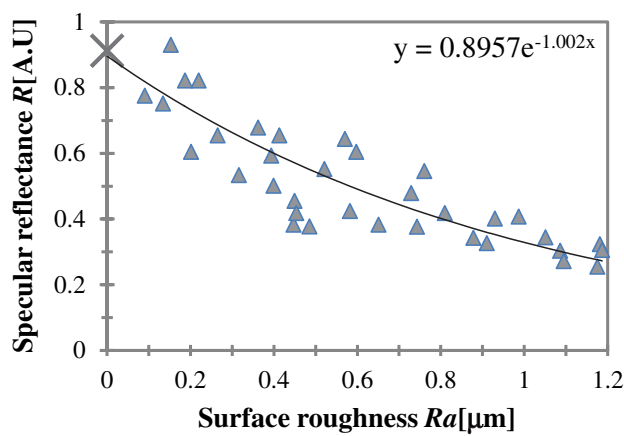

(a) Aluminum.

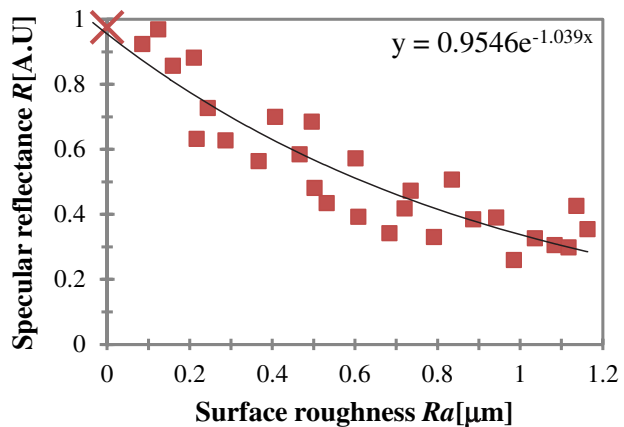

(b) Copper.

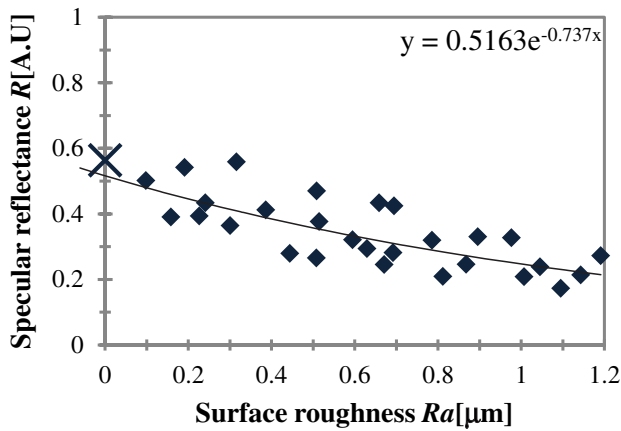

(c) Iron.

Fig. 11. (Color online) Specular reflectance using surface structures made by the AFM measurement. 


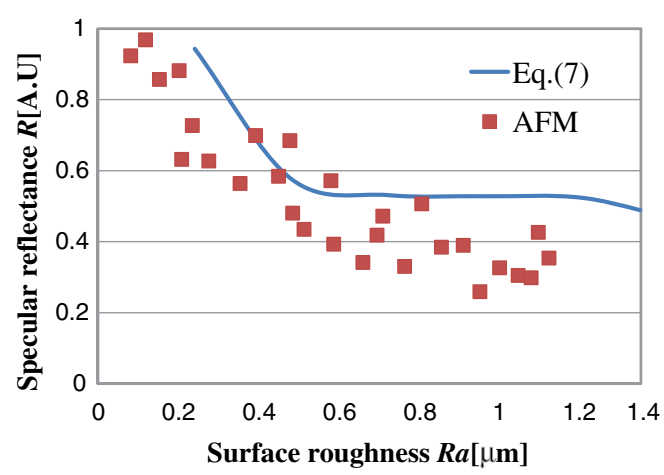

(a) Copper.

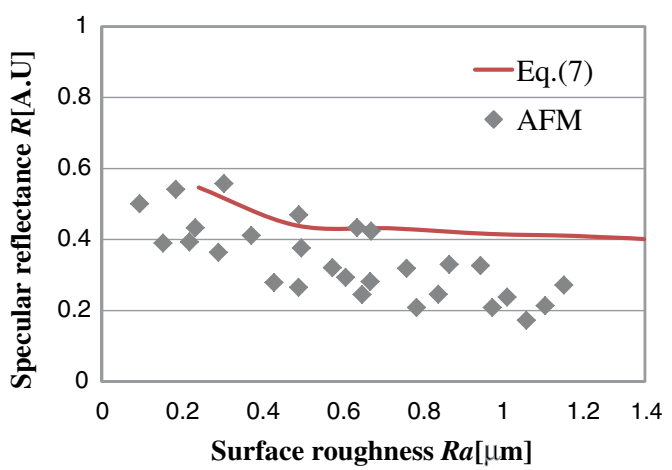

(b) Iron.

Fig. 12. (Color online) Comparison of the specular reflectance by Eq. $\underline{7}$ and AFM.

Both sides of the cylinder are faded compared to Fig. 13(b). This is because the scatter angle from a minute uneven surface is narrow, so the object light does not reach angular directions. The results in Subsection 5.A show that, the rougher the surface structure, the wider the scatter angle. Therefore, wider areas of the object can be seen in Fig. 13(c) than in Fig. 13(d). These results show that the differences of reflectance distributions caused by surface roughness are expressed.

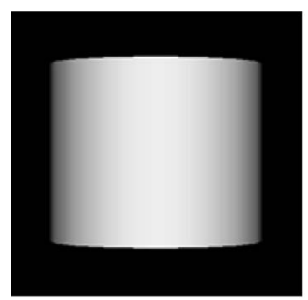

(a) Virtual image.

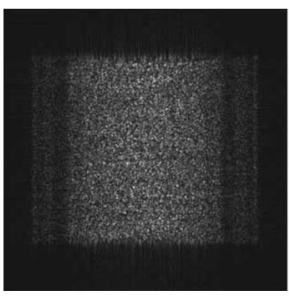

(c) $R a=1.1468[\mu \mathrm{m}]$

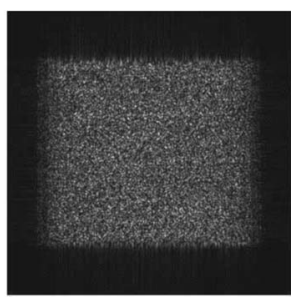

(b) Random Phase method.

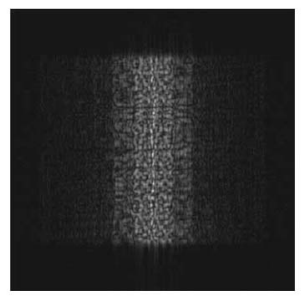

(d) $R a=0.5358[\mu \mathrm{m}]$
Fig. 13. 3D images by the imaging simulation.
Table 3. Setup Parameters for Imaging Simulations
Number of pixels

Input sampling pitch

Output sampling pitch

Wavelength
$2048 \times 2048$ [pixels]

$0.05 \times 0.05[\mu \mathrm{m}]$

$1.0 \times 1.0[\mu \mathrm{m}]$

$632[\mathrm{~nm}]$

\section{Conclusion}

We proposed a calculation method for CGHs using the FDTD method in consideration of surface roughness in order to add several reflectance distributions for each material and surface roughness to a CGH. In this method, several reflected light distributions from materials and surface structures were obtained using the FDTD method.

In a computer simulation of specular reflectance, the relations between surface roughness and specular reflectance for each material were calculated. The results showed that the differences of materials, such as dielectric and magnetic materials, not only to metallic objects, can be expressed by the proposed method. Moreover, it was confirmed that the results of surface structures made on a computer were similar to those of actual objects. Therefore, we succeeded in expressing the variation of reflectance distributions caused by differing surface roughness and materials.

In the future, the direction of incident light in the FDTD analysis should be considered. Now, our method deals with only incident light vertical to the object surface. However, when 3D models are generated, the position relation between the light source and the surface changes according to the patches of the object. Hence, a calculation method that obtains the reflected light distributions in consideration of the direction of the incident light is needed.

A calculation for displaying colors is also necessary for clearly expressing the difference of materials. The reflectivity of copper is as high as that of aluminum in the red wavelength range. However, the specular reflectance of copper decreases more rapidly in the long-wavelength side than in the red wavelength. Because there are many dispersive media that change depending on wavelength, we expect that the reality of $3 \mathrm{D}$ images will be improved by expressing the reflectivity of each wavelength.

\section{References}

1. H. Kang, T. Yamaguchi, H. Yoshikawa, S.-C. Kim, and E.-S. Kim, "Acceleration method of computing a compensated phase-added stereogram on a graphic processing unit," Appl. Opt. 47, 5784-5789 (2008).

2. Y. Ichihashi, H. Nakayama, T. Ito, N. Masuda, T. Shimobaba, A. Shiraki, and T. Sugie, "HORN-6 special-purpose clustered computing system for electroholography," Opt. Express 17, 13895-13903 (2009).

3. H. Yoshikawa, T. Yamaguchi, and R. Kitayama, "Real-time generation of full color image hologram with compact distance look-up table," in Digital Holography and Three-Dimensional Imaging, OSA Technical Digest (CD) (Optical Society of America, 2009), paper DWC4.

4. H. Sakata, and Y. Sakamoto, "Fast computation method for a Fresnel hologram using three-dimensional affine transformations in real space," Appl. Opt. 48, H212-H221 (2009). 
5. K. Matsushima, "Exact hidden-surface removal in digitally synthetic full-parallax holograms," Proc. SPIE 5742, 25-32 (2005).

6. K. Matsushima, "Computer-generated holograms for threedimensional surface objects with shade and texture," Appl. Opt. 44, 4607-4614 (2005).

7. K. Yamaguchi, T. Ichikawa, and Y. Sakamoto, "Calculation method for CGH considering smooth shading with polygon models," Proc. SPIE 7957, 795706 (2011).

8. K. S. Yee, "Numerical solution of initial boundary value problems involving Maxwell's equations in isotropic media," IEEE Trans. Antennas Propag. AP-14, 302-307 (1966).

9. J. P. Waters, "Holographic image synthesis utilizing theoretical methods," Appl. Phys. Lett. 9, 405-407 (1966).

10. J. W. Goodman, Introduction to Fourier Optics 2nd ed. (1995).

11. K. Matsuhima, H. Schimmel, and F. Wyrowski, "Fast calculation method for optical diffraction on tilted planes by use of the angular spectrum of plane waves," J. Opt. Soc. Am. A 20, 1755-1762 (2003).
12. K. Matsushima, and T. Shimobaba, "Band-limited angular spectrum method for numerical simulation of free-space propagation in far and near field," Opt. Express 17, 19662-19673 (2009).

13. R. P. Muffoletto, J. M. Tyler, and J. E. Tohline, "Shifted Fresnel diffraction for computational holography," Opt. Express 15 , 5631-5640 (2007).

14. R. Bräuer, F. Wyrowski, and O. Bryngdahl, "Diffusers in digital holography," J. Opt. Soc. Am. A 8, 572-578 (1991).

15. Y. Sakamoto, and A. Tsuruno, "A representation method for object surface glossiness in computer-generated hologram," IEICE Trans. Inf. Syst., J88-D-2, 2046-2053 (2005) (Japanese ed.).

16. K. Yamaguchi, and Y. Sakamoto, "Computer generated hologram with characteristics of reflection: reflectance distributions and reflected images," Appl. Opt. 48, H203-H211 (2009).

17. M. W. Chevalier, R. J. Luebbers, and V. P. Cable, "FDTD local grid with material traverse," IEEE Trans. Antennas Propag. 45, 411-421 (1997). 